\title{
Environmentally Friendly Magnetic Refrigeration Technology Using Ferromagnetic Gd Alloys
}

\author{
Akiko T. Saito, Tadahiko Kobayashi, Shiori Kaji, Jing Li, and Hideki Nakagome
}

\begin{abstract}
We have developed magnetic refrigerants particles of ferromagnetic Gd-based alloys and a primitive magnetic refrigeration apparatus using permanent magnets to clarify its applicability to common refrigeration. Working temperature range of the magnetic refrigeration can be controlled with the Curie temperatures of the magnetic refrigerants. Several kind of Gd-based spherically-shaped magnetic refrigerant particles have fabricated by a rotating electrode process. Operation of an active magnetic regenerative (AMR) refrigeration cycle with Gd-based magnetic refrigerant particles enabled temperature spans of more than $40{ }^{\circ} \mathrm{C}$ to be obtained at room temperature. Optimization of multi-layered structures of magnetic refrigerant materials having different Curie temperatures allowed effective enlargement of the temperature span. We achieved a cold temperature of $-11^{\circ} \mathrm{C}$ which is the lowest temperature generated by AMR cycle operation under a low magnetic field using permanent magnets. Our results show that magnetic refrigeration has application potential for new environmentally friendly refrigeration technology.
\end{abstract}

Index Terms-Magnetic refrigeration, magnetocaloric effect, entropy, Gd alloy, environmentally-friendly.

\section{INTRODUCTION}

The field of refrigeration technology faces worldwide problems, such as the high energy consumption of refrigeration systems and the significant environmental impact of refrigeration fluid emissions. The conventional vapor compression refrigeration fluids, CFCs (chlorofluorocarbons), HCFCs (hydrochlorofluorocarbons), and HFCs (hydrofluorocarbons), are ozone-depleting substances or greenhouse gases. Therefore, they have been phased out under the Montreal and Kyoto Protocols, and replaced with natural refrigerants. However, these natural refrigerants also have issues. For example, isobutene applied to the refrigerator is flammable and ammonia applied to cooling systems for the cold storage warehouse is harmful. Moreover, the power consumed in refrigeration and cooling technology amounts to

Manuscript received May 15, 2015; revised July 16, 2015. This work was partially supported financially by the Japan Science and Technology Agency, and the New Energy and Industrial Technology Development Organization.

Akiko T. Saito is with the Corporate Research and Development Center, Toshiba Corporation, 1, Komukai-Toshiba, Saiwai, Kawasaki, 212-8582, Japan. She is also with the Department of Urban Environment Systems, Chiba University, 1-33 Yayoi, Inage, Chiba 263-8522, Japan (e-mail: aki.saito@toshiba.co.jp).

Tadahiko Kobayashi and Shiori Kaji are with the Corporate Research and Development Center, Toshiba Corporation, 1, Komukai-Toshiba, Saiwai, Kawasaki, 212-8582, Japan.

Jing $\mathrm{Li}$ and Hideki Nakagome are with the Department of Urban Environment Systems, Chiba University, 1-33 Yayoi, Inage, Chiba 263-8522, Japan (e-mail: nakagome@tu.chiba-u.ac.jp). almost $30 \%$ of total electrical power use. Therefore, environmentally friendly and efficient new refrigeration technologies are required.

The Magnetocaloric effect [1] is a thermodynamically reversible phenomenon involving energy conversion between magnetic and thermal energy triggered by the application of an external magnetic field on a magnetic refrigerant. The magnetocaloric effect can be used to build a thermal cycle similar to the gas compression cycle as shown in Fig. 1; this is called magnetic refrigeration. Historically, magnetic refrigeration using paramagnetic refrigerants having large magnetic moments has been applied to the generation of extremely low temperatures difficult to achieve using a gas compression cycle [2]-[4]. In 1976, room-temperature magnetic refrigeration was achieved for the first time, when a high magnetic field applied to a ferromagnetic refrigerant of gadolinium (Gd) using a superconducting magnet [5]. Energy and environmental issues such as ozone depletion and global warming had become obvious and had been pointed as having been involved in emissions of refrigerant gases in the 1980s and the 1990s. A challenge to demonstrate 18 months continuous operation of an active magnetic regenerative (AMR) refrigeration cycle near room-temperature was achieved in 1997 [6]. This result showed the higher potential coefficient of performance of AMR refrigeration than that of the household refrigerator, although it was realized only with a high magnetic field generated by a superconducting magnet. Over the last few decades, AMR refrigeration using permanent magnets has been actively investigated [7]-[17] toward practical applications. However, there have been few reports of attainment to cool down to below zero degree by AMR refrigeration with permanent magnets.

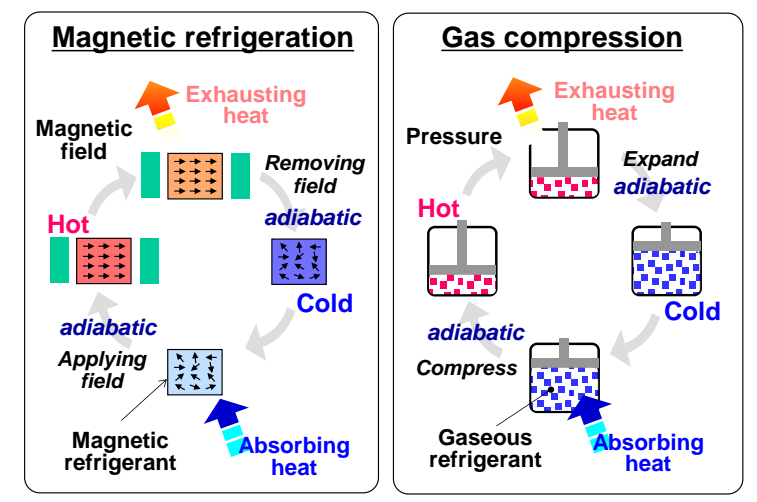

Fig. 1. Conceptual diagram of magnetic refrigeration cycle and gas compression cycle.

In this study, magnetic refrigerant particles of several kinds of Gd alloys were developed and their magnetocaloric properties were characterized. The AMR-cycle performance of the particulate Gd alloy refrigerants with layered structure 
under a low magnetic field generated by permanent magnets was investigated. Our results show that magnetic refrigeration has potential for application in cooling technology for commodities.

\section{EXPERIMENTAL}

\section{A. Experimental Apparatus and Measurement Procedures on AMR-Cycle}

The experimental apparatus used in this study is shown schematically in Fig. 2. Magnetic refrigerant particles were packed into a cylindrical container (the AMR-unit) with a filling rate of about $63 \%$, after which the cylinder was filled with heat transfer fluid. The heat transfer fluid was able to flow through the packed magnetic refrigerant particles using pistons movement attached to the top and bottom parts of the cylinder. The AMR-unit was moved into and out of the bore of a Halbach-type magnet to apply and remove a magnetic field to and from the AMR-unit. The strength of the magneticfield at the center of the bore was about 1.1 Tesla. The temperature at several points in the AMR-unit was monitored using type $\mathrm{K}$ thermocouples. The heat transfer fluid in the AMR-unit was water or $20 \%$ ethylene glycol solution. Fig. 3 represents a conceptual view of the AMR-cycle process as follows. 1) A magnetic field is applied to the AMR-unit, causing the magnetic refrigerants to warm. 2) The heat transfer fluid flows from the cold to the hot end through the magnetic refrigerants, and warm heat is transported to the hot side by the flow. 3) The magnetic field is removed from the AMR-unit, causing the magnetic refrigerants to cool down. 4) The heat transfer fluid flows from the hot to the cold end (the opposite direction to step II) through the magnetic refrigerants, and cold heat is transported to the cold side by the flow. After repeated cycles, a temperature gradient is generated in the AMR-unit. The cycle frequency was controlled by speed of the pistons movement. The AMR-unit and a Halbach-type magnet were set in a temperature- controlled room to keep the ambient temperature constant during AMR-cycle operation. The main operating conditions are given in Table I. The refrigeration properties of the AMR-cycle were examined under several conditions.

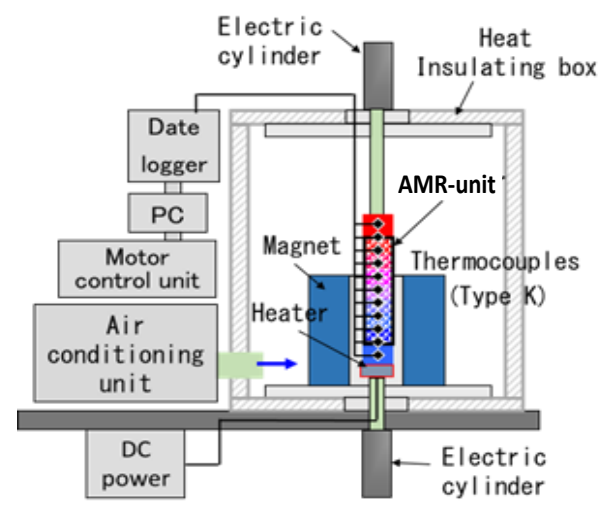

Fig. 2. Schematic of experimental apparatus.

\section{B. Material Preparation and Characterization}

Gadolinium has been considered as a promising refrigerant candidate for room-temperature magnetic refrigeration, because it shows a large entropy change around its Curie temperature $\left(T_{\mathrm{C}}\right)$ of $294 \mathrm{~K}\left(\sim 21^{\circ} \mathrm{C}\right)$. To extend the working region to lower temperature, we designed and prepared a series of ferromagnetic Gd-based alloys. The transition temperatures and magnetic properties of the obtained Gd-based alloys were confirmed by magnetic measurements using a superconducting quantum interference device (SQUID) magnetometer (Quantum Design). The Gd-based alloys were shaped into spherical particles using the rotating electrode process. Pristine spherical particles with a metallic sheen and a diameter of 0.2 to $1.2 \mathrm{~mm}$, controlled by changing the rotation speed of the mother alloy electrode, were obtained. The magnetic entropy change $\Delta S_{\mathrm{m}}$ of spherical shaped Gd alloys was estimated from magnetization curves using Eq. 1 derived from the integration of the Maxwell relations,

$$
\Delta S_{m}=\int_{H_{1}}^{H_{2}}\left(\frac{\partial M}{\partial T}\right)_{H} d H
$$

where $S_{m}$ and $M$ are the magnetic entropy and magnetization of the magnetic material, $T$ is the temperature and $H$ is the magnetic field. Several different AMR-units were packed with magnetic refrigerant particles (about $100 \mathrm{~g}$ ) of different particle size and composition, or with layers of a number of different magnetic refrigerant materials. The specifications of the AMR-units are given in Table II.

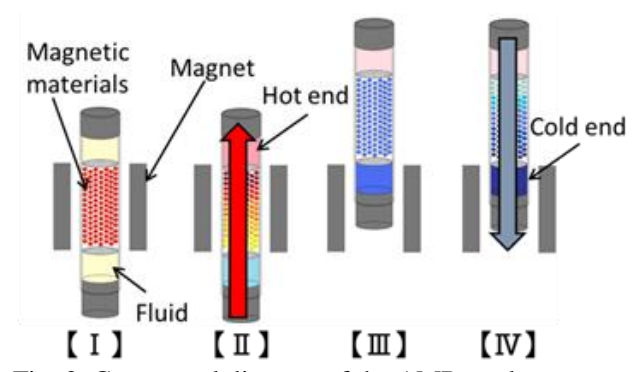

Fig. 3. Conceptual diagram of the AMR-cycle process.

TABLE I: AMR-CYCLE OPERATION CONDITIONS

\begin{tabular}{lc}
\hline \multicolumn{2}{c}{ Parameters of AMR-cycle operation and conditions } \\
\hline Ambient temperature $T_{\mathrm{i}}\left({ }^{\circ} \mathrm{C}\right)$ & $0-30$ \\
Displacement of piston $d(\mathrm{~mm})$ & $5-10$ \\
Cycle frequency $f(\mathrm{~Hz})$ & $0.1-0.4$ \\
\hline
\end{tabular}

TABLE II: THE ARRANGEMENT OF CHANNELS

\begin{tabular}{c|l|c|c}
\hline \multirow{2}{*}{ Structure } & \multicolumn{3}{|c}{ Magnetic refrigerants } \\
\cline { 2 - 4 } & \multicolumn{1}{|c}{ Composition of materials } & \multicolumn{1}{c}{$\begin{array}{c}\text { Ratio of } \\
\text { materials }\end{array}$} & $\begin{array}{c}\text { Particle size } \\
(\mathrm{m})\end{array}$ \\
\hline Single-layer A & $\mathrm{Gd}_{100}$ & 1 & 500 \\
Single-layer B & $\mathrm{Gd}_{98.5} \mathrm{Y}_{1.5}$ & 1 & $500,600,780$ \\
Double-layer & $\mathrm{Gd}_{90} \mathrm{Ho}_{10} / \mathrm{Gd}_{98.5} \mathrm{Y}_{1.5}$ & $1: 1$ & $500 / 500$ \\
Triple-layer A & $\mathrm{Gd}_{90} \mathrm{Ho}_{10} / \mathrm{Gd}_{95} \mathrm{Y}_{5} / \mathrm{Gd}_{98.5} \mathrm{Y}_{1.5}$ & $1: 1: 1$ & $500 / 500 / 500$ \\
Triple-layer B & $\mathrm{Gd}_{90} \mathrm{Ho}_{10} / \mathrm{Gd}_{95} \mathrm{Y}_{5} / \mathrm{Gd}_{98.5} \mathrm{Y}_{1.5}$ & $10: 3: 10$ & $500 / 500 / 500$ \\
\hline \hline
\end{tabular}

\section{RESULTS AND DISCUSSION}

Magnetization measurements revealed that the Curie 
temperatures of the $\mathrm{Gd}_{100-x} \mathrm{R}_{x}$ alloys $(R=Y, H o, E r)$ decreased linearly with increasing $x$ in the range of $x \leq 15$ as shown in Fig. 4. Several of these Gd-based alloys were selected and shaped into particles for application to AMR-cycle operation. Fig. 5 shows the temperature dependences of the magnetic entropy changes $\Delta S_{\mathrm{m}}$ of the Gd alloy particles used in the AMR-cycle experiments. Each of the Gd alloys showed a peak at around their $T_{\mathrm{C}}$ in the $\Delta S_{\mathrm{m}}$. The peak value of $\Delta S_{\mathrm{m}}$ for $\mathrm{Gd}_{98.5} \mathrm{Y}_{1.5}, \mathrm{Gd}_{95} \mathrm{Y}_{5}$, and $\mathrm{Gd}_{90} \mathrm{Ho}_{10}$ was equal to or higher than that of $\mathrm{Gd}$, whereas $T_{\mathrm{C}}$ was clearly lowered as intended. The refrigeration properties of the several kinds of AMR-units listed in Table II were investigated under various conditions.

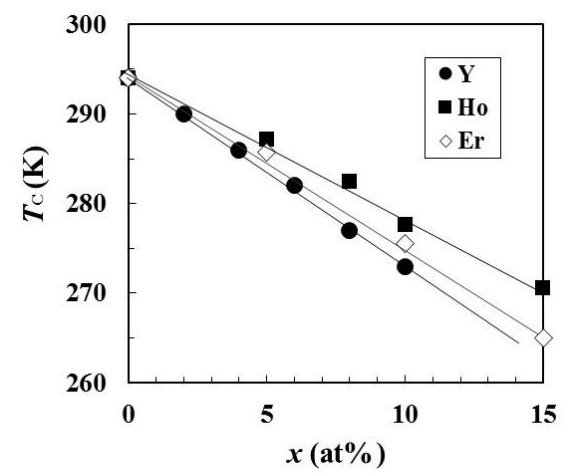

Fig. 4. Curie temperatures of $\mathrm{Gd}_{100-x} \mathrm{R}_{x}$ alloys $(\mathrm{R}=\mathrm{Y}, \mathrm{Ho}, \mathrm{Er})$.

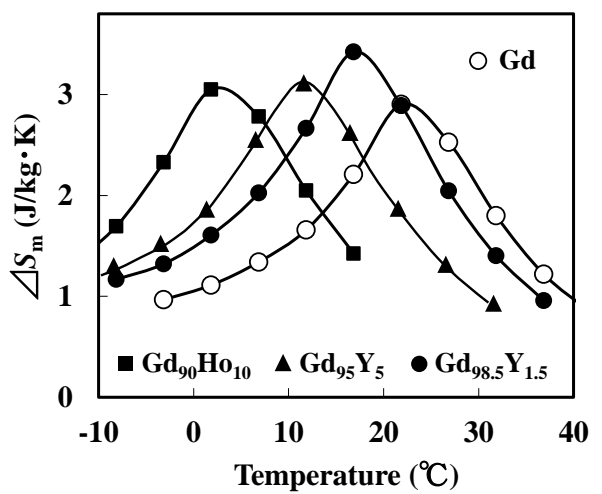

Fig. 5. Temperature dependence of magnetic entropy changes $\Delta S_{\mathrm{m}}$ in spherical particles of Gd-based alloys associated with the external field change $\Delta \mu_{0} H_{\text {ext }}=1 \mathrm{~T}$.

Fig. 6 shows a picture of main part of the refrigeration test apparatus and the AMR-unit. Thermocouples were installed into the AMR-unit, and tips of the thermocouples were placed at nine locations in the refrigerant particles, and in the heat transfer fluid at the hot and the cold ends. According to the described procedure as shown in Fig. 3, AMR-cycle was operated by up-and-down motion. Fig. 7 shows the results of AMR-cycle test using single-layered AMR-unit with Gd spherical particles at ambient temperature $T_{\mathrm{i}}=19{ }^{\circ} \mathrm{C}$. The inset in Fig. 7 demonstrates the temperature changes at the hot end and the cold end of the AMR-unit during AMR-cycle operation. The temperatures of both ends were almost the same initially and gradually changed along with the generating a temperature gradient inside of the AMR-unit by AMR-cycle operation to become saturated. The temperature difference between both ends in a steady-state, defined as the temperature span $\Delta T_{\text {span }}$, is shown in Fig. 7 as a function of AMR-cycle frequency. The temperature span increased linearly with increasing cycle frequency in the range of $0.1<f$
$<0.4$. The saturation behavior of both ends temperature is due to the balancing of generation of temperature gradient by the AMR-cycle and heat flow from the higher to the lower temperature region. Therefore, increase of cycle frequency contributed to enhancement in the generating temperature gradient that resulted in the increase of $\Delta T_{\text {span }}$.

Effects of particle size and piston displacement on $\Delta T_{\text {span }}$ were also investigated. Fig. 8 shows the particle size dependence of $\Delta T_{\text {span }}$ on the single-layered AMR-unit with $\mathrm{Gd}_{98.5} \mathrm{Y}_{1.5}$ spherical particles operating the AMR-cycle at $f$ $=0.4 \mathrm{~Hz}, T_{\mathrm{i}}=15^{\circ} \mathrm{C}$ with the parameter of piston displacement. The temperature span decreased with increasing piston displacement from $5 \mathrm{~mm}$ to $10 \mathrm{~mm}$. This is attributed to excess mixture of the heat transfer fluid in the hot-side and the cold-side of the AMR-unit. With decreasing particle size from $780 \mu \mathrm{m}$ to $500 \mu \mathrm{m}, \Delta T_{\text {span }}$ became larger and saturated at each piston displacement. The smaller the particle size, the larger the heat exchange area between the magnetic refrigerant particles and heat transfer fluid to contribute to enhance refrigeration performance. At the same time, the smaller the particle size, the larger the pressure loss in the heat transfer fluid to deteriorate the performance. Therefore, there is an optimum particle size determined by the trade-off between an increased heat exchange area and pressure loss.
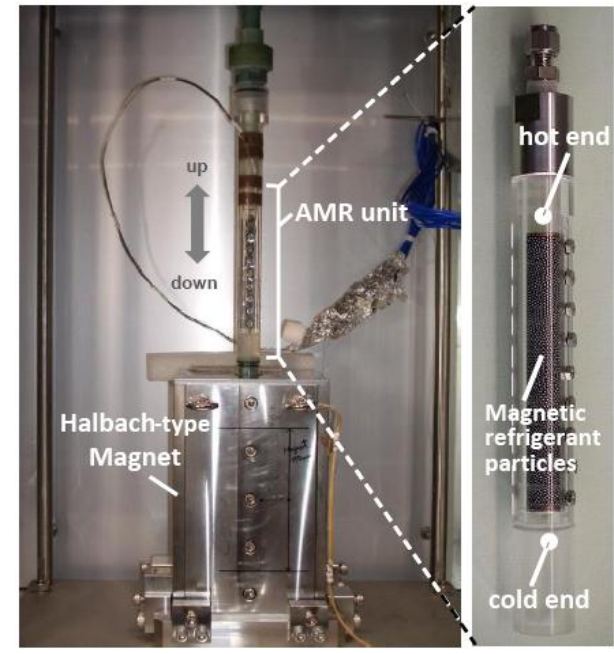

Fig. 6. Picture of the AMR-cycle apparatus and AMR-unit. The AMR-unit moves down into the bore of a Halbach-type magnet to apply a magnetic field to the magnetic refrigerant particles packed in the AMR-unit.

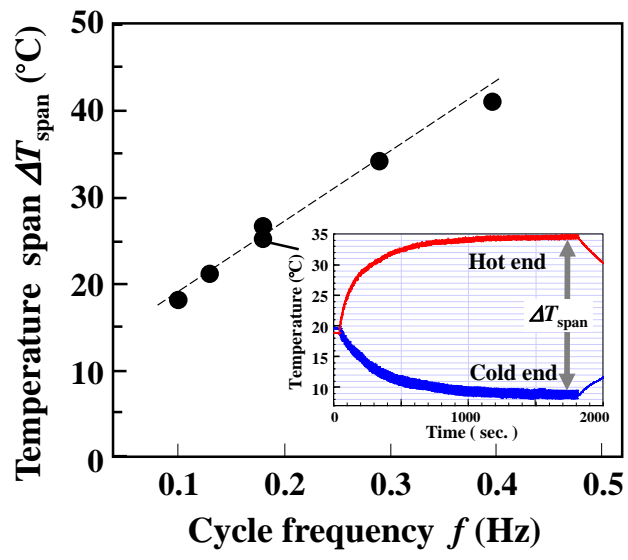

Fig. 7. Cycle frequency dependence of temperature span, $\Delta T_{\text {span, }}$, for the single-layered AMR-unit. The inset shows an example of the temperature changes occurring at both ends during AMR-cycle operation with $\mathrm{Gd}$ particles. 


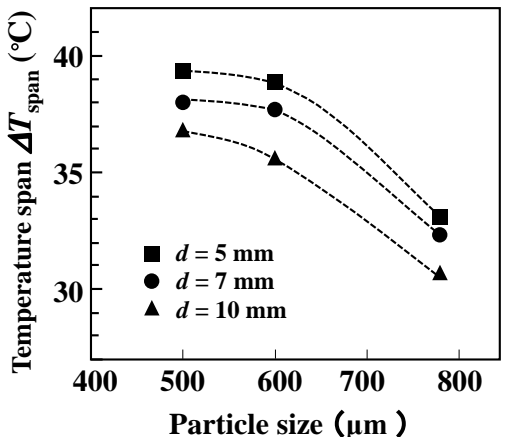

Fig. 8. Particle size dependence of temperature span, $\Delta T_{\mathrm{span}}$, on the singlelayered AMR-unit with $\mathrm{Gd}_{98.5} \mathrm{Y}_{1.5}$ spheres operating at $f=0.4 \mathrm{~Hz}$ and $T_{\mathrm{i}}=$ $15^{\circ} \mathrm{C}$, as the parameter with $d$.

Next, AMR-cycle experiments were performed with the multi-layered structures listed in Table II, which contained multiple refrigerant materials having different Curie temperature, $T_{\mathrm{C}}$. Fig. 9 shows the ambient temperature dependences of $\Delta T_{\text {span }}$ for a single-layered structure with $\mathrm{Gd}_{98.5} \mathrm{Y}_{1.5}$, double-layered structure with $\mathrm{Gd}_{90} \mathrm{Ho}_{10} / \mathrm{Gd}_{98.5} \mathrm{Y}_{1.5}$, and triple-layered structures with $\mathrm{Gd}_{90} \mathrm{Ho}_{10} / \mathrm{Gd}_{95} \mathrm{Y}_{5}$ $/ \mathrm{Gd}_{98.5} \mathrm{Y}_{1.5}$ (A and B) refrigerants. The particle size of each refrigerant was $500 \mu \mathrm{m}$, and the same cycle frequency $(f=0.4$ $\mathrm{Hz})$ and piston displacement $(d=5 \mathrm{~mm})$ were used. For the single-layered structure with $\operatorname{Gd}_{98.5} \mathrm{Y}_{1.5}$ particles, $\Delta T_{\text {span }}$ exhibited a broad peak at about $40{ }^{\circ} \mathrm{C}$, around $T_{\mathrm{i}}=10^{\circ} \mathrm{C}$. The double-layered structure, which additionally contained $\mathrm{Gd}_{90} \mathrm{Ho}_{10}$ particles, showed a larger peak at lower temperature $T_{\mathrm{i}}=5{ }^{\circ} \mathrm{C}$. The result is in accordance with the lower $T_{\mathrm{C}}$ of $\mathrm{Gd}_{90} \mathrm{Ho}_{10}$ refrigerant. The peak value of $\Delta T_{\text {span }}$ increased further in the triple-layered structures (A and $\mathrm{B}$ ), which contained $\mathrm{Gd}_{95} \mathrm{Y}_{5}$ between the $\mathrm{Gd}_{90} \mathrm{Ho}_{10}$ and $\mathrm{Gd}_{98.5} \mathrm{Y}_{1.5}$ refrigerants arranged in order of the $T_{\mathrm{C}}$. The AMR-unit with triple-layered structure B exhibited the larger $\Delta T_{\text {span }}=45^{\circ} \mathrm{C}$ at $T_{\mathrm{i}}=5{ }^{\circ} \mathrm{C}$ than that with triple-layered structure A which constructed by divided equally parts packed these three kinds of materials. In these AMR-units of triple- layered structure A and $\mathrm{B}$, the ratio of amounts of the materials were differ from each other, while the refrigerant materials and their order were the same. These results indicate that there is an optimum ratio of amounts of each refrigerant material to give the best performance in $\Delta T_{\text {span }}$.

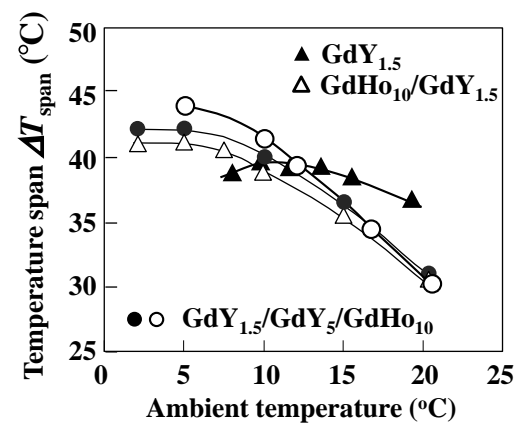

Fig. 9. Temperature span, $\Delta T_{\text {span }}$, as a function of ambient temperature for single-layered with $\mathrm{Gd}_{98.5} \mathrm{Y}_{1.5}$, double-layered with $\mathrm{Gd}_{90} \mathrm{Ho}_{10} / \mathrm{Gd}_{98.5} \mathrm{Y}_{1.5}$ and triple-layered with $\mathrm{Gd}_{90} \mathrm{Ho}_{10} / \mathrm{Gd}_{95} \mathrm{Y}_{5} / \mathrm{Gd}_{98.5} \mathrm{Y}_{1.5}$ refrigerants. Close circles and open circles represent triple-layered structure A and B, respectively.

As for the triple-layered structure, effect of the piston displacement on $\Delta T_{\text {span }}$ was investigated regarding temperature distribution in the AMR-unit. The slope of temperature inside the AMR-unit became gently with increasing piston displacement as shown in Fig. 10, this leads to the decrease in the value of $\Delta T_{\text {span. }}$. It is consistent with the results in the case of the single-layered AMR-unit mentioned above, and thought to be due to excess mixture of the heat transfer fluid in the hot-side and the cold-side of the AMR-unit. Temperature changes at the hot and the cold ends in an optimal condition with triple-layered structure B are shown in Fig. 11, where the cycle frequency $f=0.4 \mathrm{~Hz}$ and piston displacement $d=5 \mathrm{~mm}$. Cold temperature of $-11^{\circ} \mathrm{C}$ was attained which is the lowest temperature generated by AMR-cycle operation under a low magnetic field using permanent magnets.

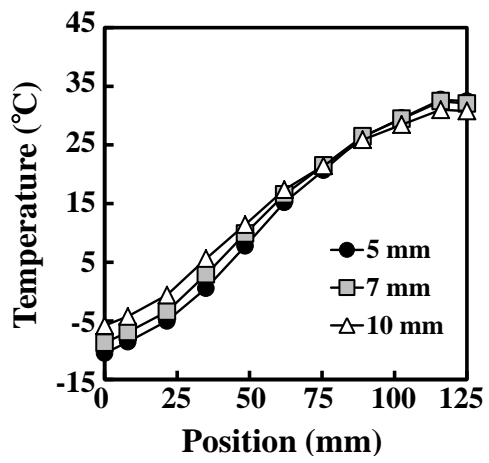

Fig. 10. Steady-state temperature distributions in the AMR-units for triple-layered structure with $\mathrm{Gd}_{90} \mathrm{Ho}_{10} / \mathrm{Gd}_{95} \mathrm{Y}_{5} / \mathrm{Gd}_{98.5} \mathrm{Y}_{1.5}$ refrigerants at different piston displacements.

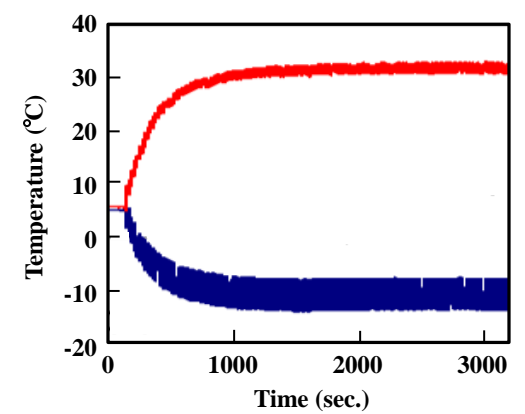

Fig. 11. Temperature changes at the hot end and the cold end of the AMR-unit with the triple-layered structure $\mathrm{B}$ constructed of $\mathrm{Gd}_{90} \mathrm{Ho}_{10} / \mathrm{Gd}_{95} \mathrm{Y}_{5} / \mathrm{Gd}_{98.5} \mathrm{Y}_{1.5}$ refrigerants during AMR-cycle operation at $f=$ $0.4 \mathrm{~Hz}$ and $T_{\mathrm{i}}=5^{\circ} \mathrm{C}$.

In terms of ambient temperature dependence, $\Delta T_{\mathrm{span}} \mathrm{S}$ of the triple-layered structure decrease rapidly in the higher temperature region compare to that in the single-layered structure, as shown in Fig. 9. To understand the different behaviors of the ambient temperature dependence of $\Delta T_{\mathrm{span}}$ of the triple-layered and single-layered structure, the internal temperature distributions in the different AMR-units were studied particularly. Fig. 12(a) and Fig. Fig. 12(b) show the steady-state temperature distributions in the AMR-units containing the single-layered structure $\mathrm{B}$ and the triple -layered structure B at different ambient temperature $T_{\mathrm{i}} \mathrm{s}$. The horizontal lines in the graphs of Fig. 12(a) and Fig. 12(b) represent the Curie temperatures of the refrigerant materials packed in different positions. The refrigerant materials located around a position corresponding to the point at the intersection of temperature curves with horizontal lines work best. Then, it is obvious that the effective volume of the working refrigerant in the triple-layered structure $\mathrm{B}$ for $T_{\mathrm{i}}=$ $5{ }^{\circ} \mathrm{C}$ was considerably larger than that in the single-layered 
structure B for $T_{\mathrm{i}}=10^{\circ} \mathrm{C}$ in the best performances of both. However, with increasing ambient temperature, the internal temperature distribution in the triple-layered structure $\mathrm{B}$ changed rapidly from preferable to mismatched, because the arrangement of refrigerant materials was optimal at $T_{\mathrm{i}}=5{ }^{\circ} \mathrm{C}$. In the case of the single-layered structure $\mathrm{B}$, the effective volume of the working refrigerant did not significantly change in the range of $7 \leq T_{\mathrm{i}} \leq 20$. This caused the ambient

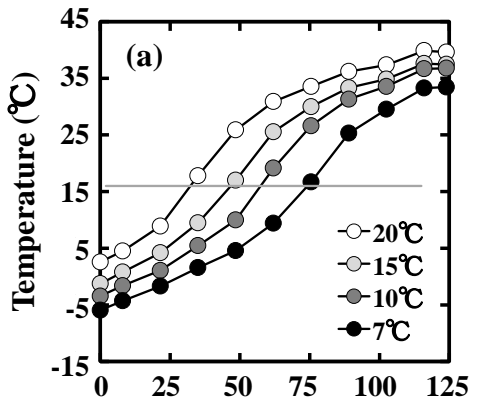

AMR position (mm) temperature dependence of $\Delta T_{\text {span }}$ in the single-layered structure to exhibit a broad peak as shown in Fig. 9. These results indicate that optimization of the arrangement of multiple refrigerant materials having different optimum working temperature according to the internal temperature distribution will realize the best refrigeration performance. However, at the same time, excess optimization will deteriorate the robustness of the performance.

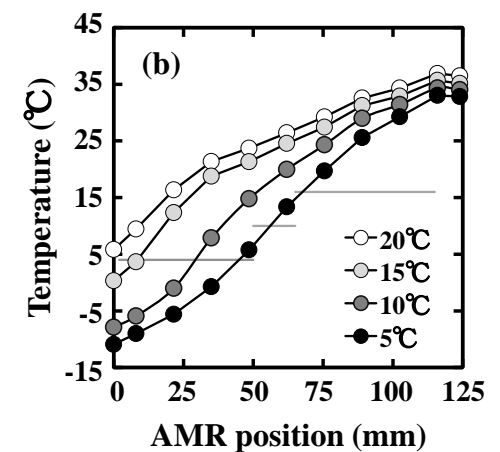

Fig. 12. Steady-state temperature distributions in the AMR-units containing (a) single-layered structure B with $\mathrm{Gd}_{98.5} \mathrm{Y}_{1.5}$, and (b) triple-layered structure B with $\mathrm{Gd}_{90} \mathrm{Ho}_{10} / \mathrm{Gd}_{95} \mathrm{Y}_{5} / \mathrm{Gd}_{98.5} \mathrm{Y}_{1.5}$ at different ambient temperatures.

\section{CONCLUSION}

Aiming towards application to practical cooling systems, we have developed a primitive magnetic refrigeration apparatus using permanent magnets to clarify the possibility of low-temperature generation such as sub-zero range from room temperature operation. Operating an AMR-cycle with Gd-based alloy magnetic refrigerant particles under a low magnetic field generated by permanent magnets, we succeeded in obtaining the temperature spans of more than $40{ }^{\circ} \mathrm{C}$ at room temperature. The temperature span was enlarged when optimized multi-layered structures of magnetic refrigerant materials having different optimum working temperatures were used. A lowest temperature of $-11^{\circ} \mathrm{C}$ was achieved. Adopting a rotary system for changing the magnetic field which applied to the magnetic refrigerant enables AMR-cycle frequency to be higher, and could improve the refrigeration performance. Although there are still many problems to be overcome, magnetic refrigeration has potential for application to new environmentally friendly refrigeration technology.

\section{REFERENCES}

[1] E. Warburg, "Magnetische untersuchungen," Ann. Phys., vol. 13, pp. $141-146,1881$

[2] P. Debye, "Einige bemerkungen zur magnetisierung bei tiefer temperatur," Ann. Phys., vol. 81, pp. 1154-1160, 1926.

[3] W. F. Giauque and I. D. P. MacDougall, "Attainment of temperatures below 1 absolute by demagnetization of $\mathrm{Gd}_{2}\left(\mathrm{SO}_{4}\right)_{8} \mathrm{H}_{2} \mathrm{O}$," Phys. Rev., vol. 43 , p. $768,1933$.

[4] D. G. Onn, H. Meyer, and J. P. Remeika, "Calorimetric study of several rare-earth gallium garnets," Phys. Rev., vol. 156, p. 663, 1967.

[5] G. V. Brown, "Magnetic heat pumping near room temperature," $J$. Appl. Phys., vol. 47, pp. 3673-3680, 1976.

[6] C. B. Zimm et al., "Description and performance of a near-room temperature magnetic refrigerator," Adv. Cryog. Engin., vol. 43, pp. 1759-1766, 1998.

[7] X. Bohigas et al., "Room temperature magnetic refrigerator using permanent magnets," IEEE Trans. Magn., vol. 36, pp. 538-544, 2000.

[8] P. Colt, D. Viallet, F. Allab, A. Kedous-Lebouc, J. M. Fournier, and J. P. Yonnet, "A magnet-based device for active magnetic regenerative refrigeration," IEEE Trans. Magn., vol. 39, pp. 3349-3351, 2003.
[9] M. Balli et al., "A pre-industrial magnetic cooling system for room temperature application," in Proc. the 4th IIF-IIR International Conference on Magnetic Refrigeration at Room Temperature, 2010, pp. 313-322.

[10] A. Tura, S. Shopfer, A. Rowe, A. Maiorino, and C. Aprea, "Heat transfer and viscous losses in micro-channel passive regenerators. Part 1 - Apparatus and experimental results," in Proc. the 4th IIF-IIR International Conference on Magnetic Refrigeration at Room Temperature, 2010, pp. 285-293.

[11] C. R. H. Bahl et al., "Development and experimental results from a 1 $\mathrm{kW}$ prototype AMR," in Proc. the 5th IIF-IIR International Conference on Magnetic Refrigeration at Room Temperature, 2012, pp. 53-60.

[12] A. Tura, A. Rowe et al., "Regenerative magnetocaloric systems: An overview of research and development activities at the university of Victoria," in Proc. the 5th IIF-IIR International Conference on Magnetic Refrigeration at Room Temperature, 2012, pp. 73-87.

[13] J. M. Gatti, C. V. Muller, G. Brumpter, and P. Haegel, "Magnetic heat pumps - Configurable hydraulic distribution for a magnetic cooling system," in Proc. the 5th IIF-IIR International Conference on Magnetic Refrigeration at Room Temperature, 2012, pp. 341-348.

[14] P. Y. Jin, J. H. Huang, H. W. Yan, and Y. Deng, "Design of an improved reciprocal room-temperature magnetic refrigerator," in Proc. the 5th IIF-IIR International Conference on Magnetic Refrigeration at Room Temperature, 2012, pp. 325-331.

[15] J. A. Lozano et al., "Experimental and numerical results of a high frequency rotating active magnetic refrigerator," in Proc. the 5th IIF-IIR International Conference on Magnetic Refrigeration at Room Temperature, 2012, pp. 373-380.

[16] J. Tusek et al., "Active magnetic regenerator (AMR) experimental test device," in Proc. the 5th IIF-IIR International Conference on Magnetic Refrigeration at Room Temperature, 2012, pp. 429-436.

[17] D. Lewandowski, "Estimating the efficiency of cooling system based on gadolinium using a simple model of heat capacity," in Proc. the 5th IIF-IIR International Conference on Magnetic Refrigeration at Room Temperature, 2012, pp. 539-547.

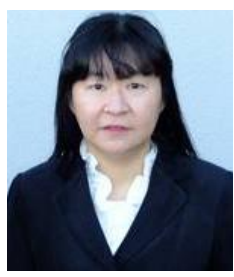

Akiko T. Saito was born in Tokyo, Japan in December 1964. She received her bachelor's degree and master's degree in physics from Hokkaido University, Sapporo, Japan in 1988 and 1990, respectively, and the doctor degree in energy engineering from Tokyo Institute of Technology, Tokyo, Japan in 1995. She is a senior research scientist at R\&D Center of Toshiba Corporation. Her main research interests are environmentally friendly techniques approached from an application of new functional material. 